\title{
The role of particle energy and pulsed particle flux in physical vapor deposition and pulsed-laser deposition
}

\author{
Stefan G. Mayr, ${ }^{\text {a) }}$ Michael Moske, ${ }^{\text {b) }}$ and Konrad Samwer ${ }^{\mathrm{c}}$ \\ Institut für Physik, Universität Augsburg, D-86135 Augsburg, Germany
}

\author{
Maggie E. Taylor and Harry A. Atwater \\ Thomas J. Watson Laboratories of Applied Physics, California Institute of Technology 128-95, Pasadena, \\ California 91125
}

(Received 9 June 1999; accepted for publication 3 November 1999)

\begin{abstract}
Surface morphology evolution of thin films generated by physical and pulsed-laser deposition depending on the incident particle energy and the pulse rate is investigated using a continuum growth model. The model includes curvature-induced surface diffusion, the Schwoebel barrier and surface atom displacement as main surface processes. The numerical solution of the model is in very good agreement with the results of kinetic Monte Carlo simulations, which also serve to estimate the continuum growth parameters, and with experimental results on thin Si films. The increase of the incident particle energy, starting from thermal energy, fundamentally influences the surface topography, changing from self-affine to self-organized morphology. (C) 1999 American Institute of Physics. [S0003-6951(99)03252-0]
\end{abstract}

The growth characteristics of thin films, primarily the surface roughness, ${ }^{1}$ e.g., epitaxial $(1 \times 1) \mathrm{Si}(001)$ growth, ${ }^{2}$ are fundamentally influenced by the energy of the deposited particles and the temporal profile of the deposition flux. These two parameters are experimentally accessible by the different film preparation techniques, such as pulsed-laser deposition (PLD), sputtering (both with a medium energy of a few $\mathrm{eV}$ to tens of $\mathrm{eV}$, pulsed or continuous temporal profile) and molecular-beam epitaxy (MBE) (thermal energy, steady-state temporal profile). These aspects are investigated by means of continuum models, ${ }^{3-10}$ of the shape:

$$
\frac{\partial h(\mathbf{x}, t)}{\partial t}=F[h(\mathbf{x}, t)]+\eta(\mathbf{x}, t) ; \mathbf{x}=(x, y),
$$

where $F[h(\mathbf{x}, t)]$ denotes all kinds of atomic processes present (assumed to be spatially isotropic-the radial average for anisotropic systems), $h(\mathbf{x}, t)$ describes the surface height profile, and $\eta$ is a spatially and temporally uncorrelated Gaussian noise. In addition to curvature induced surface diffusion, ${ }^{4,7,8}$ the Schwoebel barrier ${ }^{9,11-13}$ causes a slope-dependent modification of the average diffusion behavior. The deposition conditions (a normal particle flux is assumed) modify the surface morphology by displacements of surface atoms via kinetic energy transfer ${ }^{10,14}$ (experimentally a broad non-Maxwellian distribution with a peak at 20 $\mathrm{eV}$ and a high energy tail extending to $\approx 120 \mathrm{eV}$ in the case of $\mathrm{PLD}^{2}$ ). The macroscopic deposition rate (i.e., the time average) is assumed to be constant.

To test the validity of the model predictions, the results of a numerical solution are optimized for an agreement with a kinetic Monte Carlo model (KMC) ${ }^{15}$ and with experimental

\footnotetext{
${ }^{a)}$ Electronic mail: Stefan.Mayr@Physik.Uni-Augsburg.DE

${ }^{\text {b) }}$ Present address: Research center caesar, Friedensplatz 16, D-53111 Bonn, Germany.

${ }^{c}$ Present address: I. Physikalisches Institut, Universität Göttingen, Bunsenstr. 9, D-37073 Göttingen, Germany.
}

transmission electron microscopy (TEM) investigations of the surface morphology of $\mathrm{Si}$ on $\mathrm{Si}$ (Ref. 2) by calculating the root mean square roughness and the height-height correlation function, which shows a distinct oscillatory behavior in the case of long range surface correlations. ${ }^{16}$ The aim of this fit is to investigate which mechanisms experimentally play a dominant role, and whether and how they can be modeled, which would then allow us to use the simulations to tailor parameters for technically desired films. The continuum description applied in the following is the model for the Schwoebel effect by Politi, Elkinani, and Villain, 5,6,17 supplemented by the surface atom displacement effect: ${ }^{10,14}$

$$
\begin{aligned}
j_{S}(m)= & \frac{I l_{S} l_{D} m}{2\left(1+l_{S} / l_{D}+|m| l_{S}\right)\left(1+|m| l_{D}\right)} \\
& -\nabla\left(\frac{I l_{D}^{2}}{8\left(1+m^{2} l_{D}^{2}\right)}\right)
\end{aligned}
$$$$
F[h(x, t)]=-D_{D} \nabla^{4} h(x, t)-\nabla j_{S}(m)+C_{K} \nabla^{2} h(x, t) ;
$$

where $m$ denotes the local slope, $l_{D}$ the diffusion length (typically the width of a terrace, just before a new terrace is nucleated on top of it), $l_{S}$ the Schwoebel length (which is a measure for the asymmetry in the step edge sticking coefficients), ${ }^{5,6}$ and $I$ is the flux. The coefficient $D_{D}$ controls curvature induced surface diffusion proportional to the diffusion constant, and $C_{K}$ the atomic knockout mechanism. The magnitude of the simulation parameters were optimized for an agreement with the KMC results (with the lattice parameter as length unit, and the numbers of monolayers at constant flux as time units). Starting with linear stability considerations (e.g., Ref. 4) and the development of the surface roughness in the early stages of growth at one specific substrate temperature $\left(300^{\circ} \mathrm{C}\right)$ determines the relation of $D_{D}$ and $l_{S}, l_{D}$ and $C_{K}(I \equiv 1)$. The deposition noise intensity is chosen in agreement with the deposition flux. ${ }^{10}$ The value of 

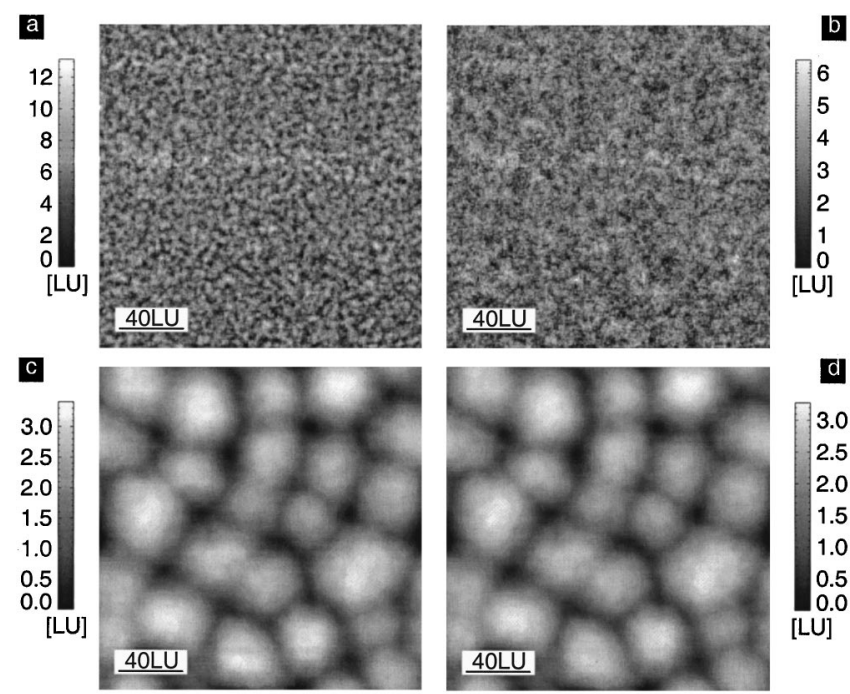

FIG. 1. Surface topographs generated by a numerical solution of the continuum model for pulsed laser deposition: The roughness is higher (a),(c) for thermal deposition than (b),(d) for energetic deposition and decreases with increasing temperature [(a),(b) $200^{\circ} \mathrm{C}$, (c),(d) $400{ }^{\circ} \mathrm{C}$ ]. At high temperature, there is hardly any difference between thermal and energetic deposition $[200 \times 200$ points, (a),(b) $\Delta t=0.001$ and (c),(d) $0.0001 ; \Delta x=1$; LU: length units].

$l_{D}$ was estimated from the $\mathrm{KMC}$ images, $l_{S}$ by a fitting to the $\mathrm{KMC}$ results and $C_{K}$ from the incident particle energy distribution together with a displacement probability $\left(C_{K}=0\right.$ in the case of thermal deposition and $C_{K}=0.38$ for energetic deposition). ${ }^{14}$ The atomic knock-out effect was assumed to be temperature independent, whereas the other parameters depend on temperature: An Arrhenius-type behavior is assumed for $D_{D}$ (with the activation energy $0.55 \mathrm{eV}$ ), ${ }^{10,15}$ and the $\mathrm{KMC}$ results determine the temperature dependence of $l_{D}$ and $l_{S}$.

The model is solved numerically for different pulsing rates and for unpulsed condition, using the Heun method ${ }^{18}$ as temporal discretization and the standard finite difference scheme $^{19}$ for spatial discretization. The effect of pulsing is built in by varying the intensity and repetition rate of the deposition noise $\eta$. While in the case of continuous noise the surface evolves continuously, in the case of pulsed deposition, the roughness generated by the pulses (turning on the noise) is smoothed out by the deterministic equation in the pulse breaks, leading to smooth hill-like structures. This can be seen from the simulated surface topographs and the experimental reflection high-energy electron diffraction (RHEED) oscillations. ${ }^{20}$ Figure 1 shows four topographs generated by numerically solving the continuum model for pulsed deposition of 20 monolayers at temperatures of 200 and $400{ }^{\circ} \mathrm{C}$ with different particle energies. Qualitatively, films produced at low temperature show a greater difference between thermal and energetic deposition, while this distinction vanishes almost completely at high temperature. From gray scale visualizations, hardly any difference between continuous deposition and film growth with different pulsing rates can be detected.

Quantitatively, the roughness $\sigma$ depicted in Fig. 2 shows a pronounced decrease with temperature in the case of thermal deposition approaching the high temperature regime with the values determined for energetic deposition, which Downloaded 18 Aug 2007 to 131.215.225.9. Redistribution subject

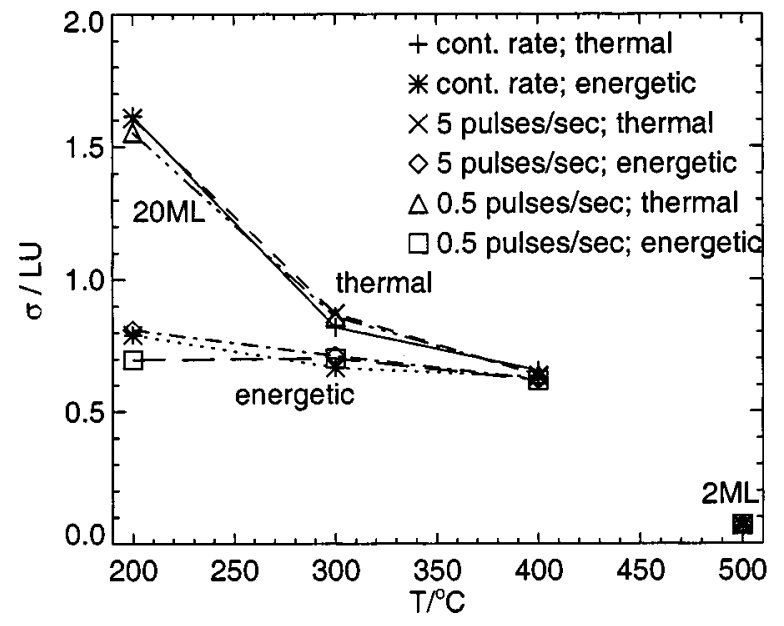

FIG. 2. RMS roughness $\sigma$ for the simulations of different film preparation techniques: With increasing temperature, $\sigma$ decreases, where the decrease for thermal deposition is stronger. In the high temperature regime, the difference between thermal and energetic deposition vanishes. For $500{ }^{\circ} \mathrm{C}$ only the deposition of two monolayers (ML) was simulated.

decrease much less. Pulsed deposition at different pulsing rates and continuous deposition differ mostly for high temperature, where the lowest pulsing rate shows the greatest smoothness on top of the hill-like structures in agreement with experimental observations ${ }^{2}$ and $\mathrm{KMC}$ simulations. ${ }^{15}$ However, the comparable approach of the high temperature roughness values takes place at a temperature about $100 \mathrm{~K}$ higher, which might be eliminated by further systematic parameter optimization.

The observation, that structure formation obviously is more significant in the case of thermal deposition, is directly confirmed by the evaluation of the height-height correlation function in Fig. 3: A distinct minimum and the second maximum are only present for thermal deposition, which is a direct consequence of the counteracting mechanisms "surface atom displacement" and "Schwoebel barrier." This suggests that for energetic deposition, the surface is much better described as self-affine. ${ }^{3}$ The surface atom displacement probability is largely temperature independent ${ }^{2,14}$ in contrast to the other mechanisms considered. They dominate

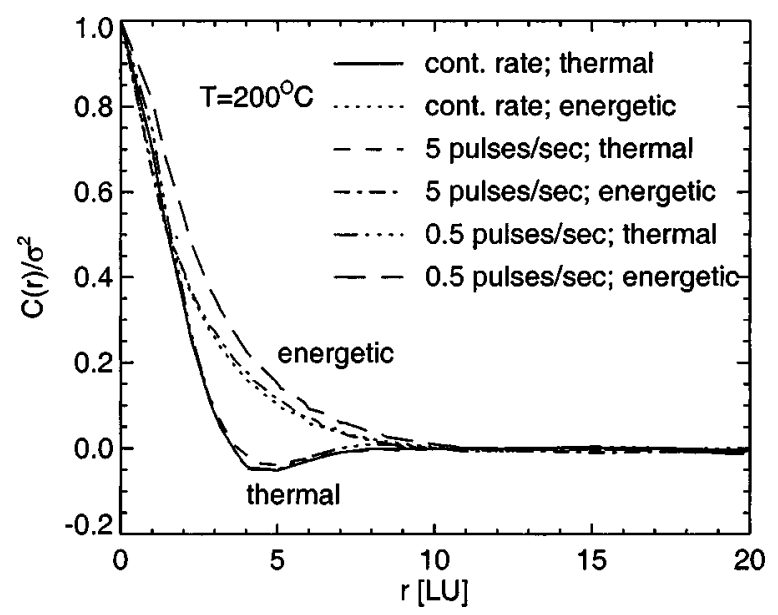

FIG. 3. Height-height correlation functions for different deposition methods: Structure formation is primarily present with thermal deposition, while it is inhibited with high energy particles. The three curves for thermal deposition are almost undistinguishable. 
the displacement effect at high temperatures, leading to pronounced structure formation in the high temperature regime. From the height-difference correlation functions, the roughness exponent ${ }^{3}$ can be determined as $\alpha \approx 0.95 \pm 0.1$ in the high temperature regime.

To conclude, the numerical solution of the continuum model applied here shows the fundamental aspects in agreement with experimental observations and KMC models: The roughness for energetic particle deposition is smaller than for thermal deposition. The difference decreases with the increasing temperature. At low temperatures, only thermal deposition shows pronounced structure formation, whereas films produced by energetic particle deposition are much better described as self-affine. The difference between pulsed deposition at different pulsing rates and continuous deposition is not significant concerning roughness for all the temperatures and pulsing rates investigated, which were chosen as parameters in accordance with the experiments.

Financial support by the DFG-Sonderforschungsbereich 438 München-Augsburg, TP A1 and the National Science Foundation is gratefully acknowledged.

${ }^{1}$ For example, see H. Sankur, W. J. Grunnig, J. DeNatale, and J. F. Flintoff, J. Appl. Phys. 65, 2475 (1989).
${ }^{2}$ M. E. Taylor, H. A. Atwater, and M. V. R. Murty, Thin Solid Films 1-2, 85 (1998).

${ }^{3}$ A. L. Barabasi and H. E. Stanley, Fractal Concepts in Surface Growth (Cambridge University Press, Cambridge, 1995).

${ }^{4}$ S. G. Mayr, M. Moske, and K. Samwer, Phys. Rev. B (in press); S. G. Mayr, Diploma thesis, Universität Augsburg, 1997.

${ }^{5}$ P. Politi and J. Villain, Phys. Rev. B 54, 5114 (1996).

${ }^{6}$ P. Politi, J. Phys. I 7, 797 (1997).

${ }^{7}$ W. W. Mullins, J. Appl. Phys. 28, 333 (1957).

${ }^{8}$ W. W. Mullins, J. Appl. Phys. 30, 77 (1959).

${ }^{9}$ M. D. Johnson, C. Orme, A. W. Hunt, D. Graff, J. Sudijono, L. M. Sander, and B. G. Orr, Phys. Rev. Lett. 72, 116 (1994).

${ }^{10}$ D. D. Vvedensky, A. Zangwill, C. N. Luse, and M. R. Wilby, Phys. Rev. E 48, 852 (1993).

${ }^{11}$ R. L. Schwoebel and E. J. Shipsey, J. Appl. Phys. 37, 3628 (1966).

${ }^{12}$ G. Ehrlich and F. G. Hudda, J. Chem. Phys. 44, 1039 (1966).

${ }^{13}$ C. J. Lanczycki and S. DasSarma, Phys. Rev. Lett. 76, 780 (1996).

${ }^{14}$ M. V. R. Murty and H. A. Atwater, Phys. Rev. B 45, 1507 (1992).

${ }^{15}$ M. E. Taylor and H. A. Atwater, Appl. Surf. Sci. 127-129, 159 (1998); M. E. Taylor, Ph.D. thesis, CALTECH, 1998.

${ }^{16}$ G. Rasigni, F. Varnier, M. Rasigni, J. P. Palmari, and A. Llebaria, Phys. Rev. B 27, 819 (1983).

${ }^{17}$ I. Elkinani and J. Villain, J. Phys. I 4, 949 (1994).

${ }^{18}$ P. E. Kloeden, E. Platen, and H. Schurz, Numerical Solution of SDE through Computer Experiments (Springer, Berlin, Heidelberg, 1991).

${ }^{19}$ W. H. Press, B. P. Flannery, S. A. Teukolsky, and W. T. Vetterling, Numerical Recipes in Pascal (Cambridge University Press, Cambridge, 1989).

${ }^{20}$ For example see, H. Karl and B. Stritzker, Phys. Rev. Lett. 69, 2939 (1992). 